\title{
End-to-End Availability Policies and Noninterference
}

\author{
Lantian Zheng Andrew C. Myers \\ Computer Science Department \\ Cornell University \\ $\{z l t$, andru\}@cs. cornell. edu
}

\begin{abstract}
This paper introduces the use of static information flow analysis for the specification and enforcement of end-toend availability policies in programs. We generalize the decentralized label model, which is about confidentiality and integrity, to also include security policies for availability. These policies characterize acceptable risks by representing them as principals. We show that in this setting, a suitable extension of noninterference corresponds to a strong, endto-end availability guarantee. This approach provides a natural way to specify availability policies and enables existing static dependency analysis techniques to be adapted for availability. The paper presents a simple language in which fine-grained information security policies can be specified as type annotations. These annotations can include requirements for all three major security properties: confidentiality, integrity, and availability. The type system for the language provably guarantees that any well-typed program has the desired noninterference properties, ensuring confidentiality, integrity, and availability.
\end{abstract}

\section{Introduction}

Although availability is often considered one of the three key aspects of information security (along with confidentiality and integrity), availability assurance has been largely divorced from other security concerns. This paper starts to bridge the gap by giving a single, common framework for reasoning about confidentiality, integrity, and availability.

The first part of this framework is a language for specifying confidentiality, integrity, and availability policies. This policy language extends the decentralized label model [21], and thus is able to describe security policies involving mutually distrusting principals.

The second part of the framework is a semantics for the policy language, which characterizes precisely what it means for a system to enforce a policy. In the context of con- fidentiality and integrity, end-to-end security policies have generally been interpreted as information flow policies requiring that the system obey noninterference. As this paper shows, availability policies too can be interpreted as requiring a form of noninterference.

The third part of the framework is a static program analysis for enforcing confidentiality, integrity, and availability policies. Previous work has shown that it is possible to enforce end-to-end confidentiality and integrity properties by static, compile-time analysis of program text (for a survey see [24]). What is new here is a demonstration that the same approach applies to availability: an availability analysis can be expressed in tractable form as a programming language type system that also enforces confidentiality and integrity.

The paper is structured as follows. Section 2 presents the new policy language for expressing requirements for availability, integrity, and confidentiality. Section 3 instantiates this label system as program annotations in a simple programming language. Section 4 uses the operational semantics of the language to express trace-based security properties that correspond to availability, integrity, and confidentiality policies. Section 5 gives a type system for this programming language and states the corresponding security theorem: well-typed programs are semantically secure (see the appendix for proofs). Section 6 extends the simple programming language to express richer notions of availability and also to describe some aspects of distributed systems. Section 7 discusses related work, and Section 8 concludes.

\section{Availability policies}

We begin by precisely defining what is meant by "availability"; then we define an expressive policy language for availability, and we demonstrate the policy language can be used for confidentiality and integrity too.

\subsection{Availability}

A system output is considered to be available if it will be produced eventually. The output does not have to be 
correct-that is the province of integrity.

There are two common ways to specify availability. The first approach is to quantify system reliability using measurable criteria, such as the failure probability or the MTTF/MTTR (mean time to fail / mean time to recover) ratio [27]. The second approach is to specify failure factors (factors that could cause the system to fail), for example, the minimum number of host failures needed to bring down the system [25]. We adopt this second approach here.

The above description of availability glosses over another aspect of availability: timeliness. How soon does an output have to occur in order to be considered to be available? For real-time services, there may be hard time bounds beyond which a late output is useless. Reasoning about how long it takes to generate an output adds considerable complexity, so for now let us consider an output to be available if it arrives eventually. Section 6 presents an extension to this framework that supports reasoning about timeliness.

\subsection{Failures and principals}

We assume that the unavailability of a system output is attributed to a failure. There are many kinds of possible failures: for example, hardware failures such as losing power, software failures such as subversion by an attacker, and human failures such as a user who provides incorrect or even malicious inputs. Our goal is a policy language that can describe all these kinds of failures and how the availability of the system should be affected by them.

We consider a failure to be the malfunction of a principal, an entity that may affect the behavior of a system. Therefore, a failure can be denoted by the responsible principal. For some failures, the corresponding principal is simply an abstract name, which might represent hardware, users, attacks or defense mechanisms, as shown in the following examples:

- power: the main power supply of a system, whose failure may bring down the entire system.

- root: the "superuser", which has the ability to control (or shut down) a system, and to act on behalf of users.

- $\mathrm{DDoS}_{1000}$ : a distributed denial of service attack launched from 1000 machines. This principal can be used to specify the availability of a system that tolerates DDoS attacks launched from fewer than 1000 machines.

- puzzle: the puzzle generated by a puzzle-based defense mechanism [13] for DoS attacks. This principal fails if attackers can feasibly solve the puzzle and launch DoS attacks successfully.

More complex failure scenarios are described by using composite principals [1]. For example, suppose that there is a principal ups representing a back-up power supply. And to make the system unavailable, both power and ups need to fail. This joint failure is represented by a composite principal given by the conjunction power $\wedge$ ups.

More generally, principals $p$ may be constructed using conjunction and disjunction operators $\wedge$ and $\vee$ :

$$
p::=a\left|p_{1} \wedge p_{2}\right| p_{1} \vee p_{2}
$$

The notation $a$ is an abstract name representing a principal. The composite principal $p_{1} \wedge p_{2}$ represents a joint failure factor: $p_{1} \wedge p_{2}$ fails only if both $p_{1}$ and $p_{2}$ fail. Another constructor $\vee$ is used to construct a group (disjunction): principal $p_{1} \vee p_{2}$ represents a failure that happens if either $p_{1}$ or $p_{2}$ fails. For example, the principal root $\vee$ power can make a system fail if the superuser and the power supply each can cause the failure.

To demonstrate the expressiveness of this principal language, we specify the availability of a quorum system [17]. A quorum system is a collection $\left\{Q_{1}, \ldots, Q_{n}\right\}$ of sets (quorums) of hosts, every two of which intersect. A quorum system is available as long as there is some quorum in which no hosts fail. Therefore, a quorum system cannot tolerate the failure of a set of hosts $B$ such that for every quorum $Q_{i}, B \cap Q_{i}$ is not empty. Thus, if the principal $h$ represents a host, availability of a quorum system can be specified by the principal $\bigvee_{B \mid \forall Q_{i} . B \cap Q_{i} \neq \emptyset}\left(\bigwedge_{h \in B} h\right)$.

\subsection{Principal hierarchy}

We write $p_{1} \succeq p_{2}$ if the principal $p_{1}$ acts for another principal $p_{2}$-that is, $p_{1}$ has all the powers of $p_{2}$ and is at least as trustworthy [21]. Interpreting principals as failure factors, this means the failure of $p_{1}$ is worse than the failure of $p_{2}$ (or the same). The acts-for relation is useful for analyzing availability, because $p_{1} \succeq p_{2}$ means that the availability represented by $p_{1}$ is at least as high as the availability represented by $p_{2}$. For example, if hosts $h_{1}$ and $h_{2}$ are two principals, then $h_{1} \wedge h_{2} \succeq h_{1}$ holds because $h_{1}$ fails if both $h_{1}$ and $h_{2}$ fail. And information with the availability $h_{1} \wedge h_{2}$ also achieves the availability $h_{1}$, because if $h_{1}$ does not fail, $h_{1} \wedge h_{2}$ does not fail.

The acts-for relation between principals creates a principal hierarchy $\mathcal{H}$, an ordering (actually, a pre-order) on the set of principals. By the definition of acts-for, a principal hierarchy must satisfy the following deductive rules:

$$
\begin{aligned}
& p_{1} \wedge p_{2} \succeq p_{1} \quad \frac{p_{1} \succeq p_{2} p_{2} \succeq p_{3}}{p_{1} \succeq p_{3}} \quad \frac{p_{1} \succeq p_{2}}{p_{1} \succeq p_{2} \vee p_{3}} \\
& \frac{p_{1} \succeq p_{3} \quad p_{2} \succeq p_{3}}{p_{1} \vee p_{2} \succeq p_{3}} \quad \frac{p_{1} \succeq p_{2} \quad p_{1} \succeq p_{3}}{p_{1} \succeq p_{2} \wedge p_{3}}
\end{aligned}
$$




\subsection{Owned policies}

An end-to-end availability policy specifies the availability that a user requires of a system input or output. In this work, availability is specified as a principal representing a failure factor. Accordingly, an availability policy has the form $u: p$, where principal $u$ is the policy owner (the user who specifies the policy), and principal $p$ represents the required availability. For example, if Alice specifies the availability policy Alice : $h_{1} \wedge h_{2}$ on one of her files, it means that Alice requires the file to be available if hosts $h_{1}$ and $h_{2}$ do not both fail.

In general, security (including availability) rests on assumptions. In particular, the enforcement of a policy owned by user $u$ is contingent on the assumptions made by $u$. For example, system security commonly depends on a trusted computing base (TCB). If the assumption that the TCB is trustworthy is false, security may not be enforced. In a system with mutual distrust, such as a distributed system crossing administrative domains, different users might assume different components of the system trustworthy. Thus it is important to specify policy owners explicitly to indicate whose assumptions are relevant to policy enforcement.

We build on the decentralized label model (DLM) [21], which applies the notion of policy ownership to confidentiality and integrity. In the DLM, a confidentiality or integrity policy has the form $u: p_{1}, \ldots, p_{n}$, meaning that $u$ allows only principals $p_{1}, \ldots, p_{n}$ to read or update the information protected by the policy. Using disjunctive principals, the policy $u: p_{1}, \ldots, p_{n}$ can be written in the form $u: p_{1} \vee \ldots \vee p_{n}$, just like an availability policy. Furthermore, for each security property (confidentiality, integrity or availability), a policy $u: p$ can be interpreted as an assumption by $u$ that $p$ does not fail. A confidentiality policy $u: p$ means that $u$ requires the data will remain confidential as long as $p$ does not fail to keep it confidential. For integrity, $u$ requires the data will have integrity unless $p$ fails to provide correct data. As an availability policy, it says that $u$ requires that the data is available if $p$ does not fail.

Based on this commonality, we can separate a notion of owned policies from the security properties these policies apply to. Let $\pi$ abstractly represent a security property of the system; it may be a confidentiality, integrity, or availability property. Formally, we treat $\pi$ as an abstract proposition that is true if the corresponding security property holds, and false otherwise. In general, if the policy $u: p$ is applied to a security property $\pi$, it means that $u$ requires $\pi$ to hold if $p$ does not fail.

Treating owned policies separately from the underlying, abstract security properties is useful for two reasons. First, it enables a uniform semantics for security policies. Second, it may in general be infeasible to formally specify or analyze what it means for a security property to hold, particu- larly if the security violation might occur outside the computing system; some form of abstraction is needed. This abstraction does not create a problem for security enforcement as long as the dependencies between security properties induced by a computing system can be analyzed precisely.

\subsection{Policy semantics}

Whether the policy $u: p$ is applied to confidentiality, integrity, or availability properties, it corresponds to two security assumptions: that $p$ does not fail, and the assumptions made by $u$ are true.

These assumptions can be formalized as a proposition $\sigma$ using the following syntax:

$$
\sigma::=o k p\left|\sigma_{1} \wedge \sigma_{2}\right| \sigma_{1} \vee \sigma_{2}
$$

where ok $p$ means that principal $p$ does not fail. The properties of the acts-for relation can be captured formally using these propositions. If $p_{1}$ acts for $p_{2}$, this means the failure of $p_{1}$ implies the failure of $p_{2}$ :

$$
\text { ok } p_{2} \Rightarrow \text { ok } p_{1} \text { iff } p_{1} \succeq p_{2}
$$

Consequently, composite principals satisfy the following conditions:

$$
\begin{array}{lll}
\text { ok } p_{1} \vee \mathrm{ok} p_{2} & \text { iff } & \mathrm{ok}\left(p_{1} \wedge p_{2}\right) \\
\mathrm{ok} p_{1} \wedge \mathrm{ok} p_{2} & \text { iff } & \mathrm{ok}\left(p_{1} \vee p_{2}\right)
\end{array}
$$

In addition, we assume there exists an assumption configuration $\Sigma$ that maps each principal $u$ to its assumptions $\sigma=\Sigma(u)$. In general, if $u_{1} \succeq u_{2}$, then any assumption made by $u_{1}$ is considered an assumption made by $u_{2}$. Consequently, $\Sigma$ must satisfy the following condition:

$$
u_{1} \succeq u_{2} \quad \Rightarrow \quad\left(\Sigma\left(u_{2}\right) \Rightarrow \Sigma\left(u_{1}\right)\right)
$$

A security policy can be given a formal semantics in terms of these propositions. Using brackets $\llbracket \cdot \rrbracket$ to indicate the semantic function, the meaning of a policy $u: p$ is:

$$
\llbracket u: p \rrbracket=\Sigma(u) \wedge o k p
$$

Suppose a policy $P$ is applied to a security property $\pi$. Then the meaning of the policy is a characterization of when the property is guaranteed to hold. To enforce the policy is to guarantee $\pi$ under the assumption that $\llbracket P \rrbracket$ is true, that is, to ensure $\llbracket P \rrbracket \Rightarrow \pi$.

Consider the example of enforcing the availability policy Alice: $h_{1} \wedge h_{2}$ on Alice's file. The goal is to ensure that the file is available under the assumption that $\Sigma($ Alice $) \wedge$ $o k\left(h_{1} \wedge h_{2}\right)$ is true. Therefore, one way to enforce the policy is to replicate the file on hosts $h_{1}$ and $h_{2}$ because ok $\left(h_{1} \wedge\right.$ $h_{2}$ ) means that $h_{1}$ and $h_{2}$ cannot both fail, which ensures that at least one host is available to serve accesses to the file. Moreover, if $\Sigma$ (Alice) implies ok $h_{3}$, storing the file on $h_{3}$ is another way to enforce the policy. 


\subsection{Dependency analysis and policy ordering}

A system processes inputs and produces outputs, creating dependencies between security properties of those inputs and outputs. Such dependencies capture influences of the system on security and induce constraints on security policies. For example, consider a system running the following pseudo-code:

while $(i>0)$ skip;

send $i$ to $o$;

This program sends the input $i$ to the output $o$ if the value of $i$ is not positive. Otherwise, the program diverges, so the output is unavailable. Thus, the availability of $o$ depends on the integrity of $i$. For simplicity, suppose there is only one policy applied to these properties, and let $A_{o}$ represent the availability policy of $o$, and $I_{i}$ represent the integrity policy of $i$. Then $\llbracket A_{o} \rrbracket \Rightarrow \llbracket I_{i} \rrbracket$ must hold in order to enforce $A_{o}$. If $\llbracket A_{o} \rrbracket \nRightarrow \llbracket I_{i} \rrbracket$, then $\llbracket A_{o} \rrbracket$ and $\neg \llbracket I_{i} \rrbracket$ may both hold. In this case the integrity of $i$ is not guaranteed, and the program may compromise the availability of $o$. But this violates the availability policy $A_{o}$ because $\llbracket A_{o} \rrbracket$ holds.

In general, given two security properties $\pi_{1}$ and $\pi_{2}$, and their policies $P_{1}$ and $P_{2}$, if $\pi_{1}$ depends on $\pi_{2}$, then $\llbracket P_{1} \rrbracket \Rightarrow \llbracket P_{2} \rrbracket$ must hold in order to enforce $P_{1}$. The constraint $\llbracket P_{1} \rrbracket \Rightarrow \llbracket P_{2} \rrbracket$ corresponds to a natural ordering on the two policies: $P_{2}$ is at least as strong as $P_{1}$, written $P_{1} \leq P_{2}$, meaning that for any configuration $\Sigma$ and any security property $\pi, P_{1}$ is enforced on $\pi$ if $P_{2}$ is enforced on $\pi$. It is clear that $P_{1} \leq P_{2}$ is equivalent to $\forall \Sigma$. $\llbracket P_{1} \rrbracket \Rightarrow \llbracket P_{2} \rrbracket$. The quantification over $\Sigma$ ensures that analyses based on the policy ordering are insensitive to $\Sigma$.

From the semantics of policies and formulas (1) and (2) in Section 2.5, the following rule for ordering policies immediately follows:

$$
[C P] \quad \frac{u_{2} \succeq u_{1} \quad p_{2} \succeq p_{1}}{u_{1}: p_{1} \leq u_{2}: p_{2}}
$$

\subsection{Combining owned policies}

In general, different principals may have different security requirements. It is convenient to incorporate the security policies of several principals into one entity so that they can be analyzed and manipulated together. This is accomplished by writing a set of policies $\beta=\left\{P_{1}, \ldots, P_{n}\right\}$, where each $P_{i}$ is an owned policy $u_{i}: p_{i}$ applied to the same security property.

A combined policy $\beta$ is enforced if and only if all the policies in $\beta$ are enforced. As a result, the security assumption described by $\beta$ must be weaker than or equal to the security assumptions described by policies in $\beta$. Therefore, the semantics of $\beta$ is the proposition $\llbracket \beta \rrbracket=\bigvee_{P \in \beta} \llbracket P \rrbracket$. Just as with simple policies, combined policy $\beta_{2}$ is as strong as combined policy $\beta_{1}$, written $\beta_{1} \leq \beta_{2}$, if $\forall \Sigma$. $\llbracket \beta_{1} \rrbracket \Rightarrow \llbracket \beta_{2} \rrbracket$. From the semantics, the $\leq$ ordering on policies can be lifted up to an ordering on combined policies by the following rule:

$$
\frac{\forall P \in \beta_{1} \cdot \exists P^{\prime} \in \beta_{2} . P \leq P^{\prime}}{\beta_{1} \leq \beta_{2}}
$$

Importantly, the set of all the combined policies form a lattice with the following join $(\sqcup)$ and meet $(\sqcap)$ operations:

$$
\begin{aligned}
& \beta_{1} \sqcup \beta_{2}=\beta_{1} \cup \beta_{2} \\
& \beta_{1} \sqcap \beta_{2}=\left\{u_{1} \vee u_{2}: p_{1} \vee p_{2} \mid u_{1}: p_{1} \in \beta_{1} \wedge u_{2}: p_{2} \in \beta_{2}\right\}
\end{aligned}
$$

The join and meet operations are sound with respect to the policy semantics, because it is easily shown that $\llbracket \beta_{1} \sqcup \beta_{2} \rrbracket=$ $\llbracket \beta_{1} \rrbracket \vee \llbracket \beta_{2} \rrbracket$ and $\llbracket \beta_{1} \sqcap \beta_{2} \rrbracket=\llbracket \beta_{1} \rrbracket \wedge \llbracket \beta_{2} \rrbracket$.

Having a lattice of policies supports static program analysis [7]. For example, consider an addition expression $e_{1}+$ $e_{2}$. Let $A\left(e_{1}\right)$ and $A\left(e_{2}\right)$ represent the availability policies of the results of $e_{1}$ and $e_{2}$. Since the result $e_{1}+e_{2}$ is available if and only if the results of $e_{1}$ and $e_{2}$ are both available, we have $A\left(e_{1}+e_{2}\right) \leq A\left(e_{1}\right)$ and $A\left(e_{1}+e_{2}\right) \leq$ $A\left(e_{2}\right)$. Because the policies form a lattice, $A\left(e_{1}+e_{2}\right)=$ $A\left(e_{1}\right) \sqcap A\left(e_{2}\right)$ is the least restrictive availability policy we can assign to the result of $e_{1}+e_{2}$. Dually, if $C\left(e_{1}\right)$ and $C\left(e_{2}\right)$ are the confidentiality policies of $e_{1}$ and $e_{2}$, then $C\left(e_{1}\right) \leq C\left(e_{1}+e_{2}\right)$ and $C\left(e_{2}\right) \leq C\left(e_{1}+e_{2}\right)$. The least restrictive confidentiality policy that can be assigned to the result of $e_{1}+e_{2}$ is $C\left(e_{1}\right) \sqcup C\left(e_{2}\right)$.

\subsection{Security labels}

In general, a system will need to simultaneously enforce policies for confidentiality, integrity, and availability of the information it manipulates. These policies can be applied to information as security labels. A label $\ell$ is written as a triple $\left\langle\beta_{C}, \beta_{I}, \beta_{A}\right\rangle$, where $\beta_{C}$ represents the (possibly combined) policy for confidentiality, $\beta_{I}$ represents the integrity policy, and $\beta_{A}$ represents availability. The notations $C(\ell)$, $I(\ell)$, and $A(\ell)$ represent the confidentiality, integrity, and availability components of $\ell$.

For example, suppose expression $e_{1}$ has a security label $\ell_{1}$, and $e_{2}$ has label $\ell_{2}$. Then $e_{1}+e_{2}$ has a label $\left\langle C\left(\ell_{1}\right) \sqcup\right.$ $\left.C\left(\ell_{2}\right), I\left(\ell_{1}\right) \sqcap I\left(\ell_{2}\right), A\left(\ell_{1}\right) \sqcap A\left(\ell_{2}\right)\right\rangle$.

\section{Applying policies to computation}

In this paper, a system is modeled by a program with which users (including attackers) can interact only by affecting its inputs and observing its outputs. Security policies, including confidentiality, integrity and availability policies, are specified on the inputs and outputs of a program. This section shows this approach with a simple programming language. 


\subsection{Security model}

This section introduces two security assumptions that enables enforcing security policies in a system by noninterference. One assumption specifies which policies are already enforced, and the other designates the power of attackers.

Our goal is to ensure that a program does not allow attackers to violate its security policies at run time. A program itself has no influence on how its inputs are generated or how its outputs are used by external users. Therefore, a program is not responsible for the enforcement of the integrity and availability policies of its inputs, or the confidentiality policies of its outputs. Therefore, we have the following security assumption:

SA1 Confidentiality policies specified on inputs, and integrity and availability policies specified on outputs are already enforced.

We are interested in the security violations that may be caused by attackers, and we assume that the power of an attacker is limited to affecting the inputs and observing the outputs of the system. This leads to our second security assumption:

SA2 If the integrity or availability of an output is compromised by attackers, it is because the integrity or availability of some input is compromised by attackers.

By (SA1) and (SA2), the availability policy $A_{o}$ specified on an output $o$ can be enforced by a noninterference property: the availability of the output $o$ is not interfered with by the availability of any input whose availability policy is not as strong as $A_{o}$, or by the value of any input whose integrity policy is not as strong as $A_{o}$.

Indeed, suppose the output $o$ is made unavailable by attackers. By (SA2), it is because the availability or integrity of some input $i$ is compromised by attackers. Without loss of generality, suppose the availability of $i$ is compromised. Let $A_{i}$ be the availability policy of $i$. By (SA1), $A_{i}$ is enforced, which, plus the unavailability of $i$, implies that $\llbracket A_{i} \rrbracket$ is false. By the noninterference property, we have $A_{o} \leq A_{i}$, which implies $\llbracket A_{o} \rrbracket \Rightarrow \llbracket A_{i} \rrbracket$. Thus, $\llbracket A_{o} \rrbracket$ is false because $\llbracket A_{i} \rrbracket$ is false. Therefore, the unavailability of $o$ implies that $\llbracket A_{o} \rrbracket$ is false. In other words, if $\llbracket A_{o} \rrbracket$ is true, then $o$ must be available, which means that $A_{o}$ is enforced.

One advantage of enforcing an availability policy by noninterference is to avoid proving that a program will eventually produce an output, which generally amounts to solving the halting problem.

\subsection{The Aimp programming language}

It is well known that confidentiality and integrity policies can be enforced by static program analyses that ver-

$$
\begin{array}{rlll}
\text { Values } & v & := & n \mid \text { none } \\
\text { Expressions } & e & := & n|! m| e_{1}+e_{2} \\
\text { Statements } & s \quad:= & \text { skip }|m:=e| s_{1} ; s_{2} \\
& \mid \begin{array}{l}
\text { if } e \text { then } s_{1} \text { else } s_{2} \\
\text { while } e \text { do } s
\end{array}
\end{array}
$$

Figure 1. Syntax of Aimp

ify whether a program satisfies a noninterference property $[30,11,32]$. Since availability policies also correspond to a noninterference property in our security model, a static program analysis can be used to determine whether a system satisfies these policies. We now demonstrate this approach by formally representing the system as a program written in a security-typed imperative language called Aimp.

The Aimp language is a basic imperative language with assignments, sequential composition, conditionals and loops. What distinguishes Aimp from other security-typed imperative languages [30] is the value none, which is used to represent unavailability: a value is unavailable if and only if it is none. Intuitively, there are three rules on using the value none:

- The value none cannot appear in program code.

- The result of expression $e$ is none if the evaluation of $e$ depends on none.

- The execution of a statement gets stuck if the execution depends on none.

A program of Aimp is just a statement, and the state of a program is captured by a memory $M$ that maps memory references (memory locations) to values. We assume that memory is observable to users, so memory references can be used to represent I/O channels. A reference representing an input is called an input reference. If the value of an input reference is none, then the corresponding input is unavailable. Similarly, a reference representing an output is called an output reference. Suppose $m$ is an output reference, then the corresponding output becomes available if $m$ is assigned an integer value. An unassigned output reference represents an output still expected by users.

The syntax of Aimp is shown in Figure 1. Let $m$ range over memory locations. In Aimp, values include integer $n$, and none. Expressions include integer $n$, dereference expression $! m$, and addition expression $e_{1}+e_{2}$. Note that none is not a valid expression so that it cannot appear in program text. Statements include the empty statement skip, the assignment statement $m:=e$, sequential composition $s_{1} ; s_{2}$, if and while statements.

Let $\beta$ range over a lattice $\mathcal{L}$ of base labels, such as policies as defined in Section 2. The top and bottom elements of $\mathcal{L}$ are represented by $T$ and $\perp$, respectively. The syntax 
for types in Aimp is shown as follows:

$$
\begin{array}{rrrl}
\text { Base labels } & \beta & \in \\
\text { Labels } & \ell, p c & := & \left\langle\beta_{C}, \beta_{I}, \beta_{A}\right\rangle \\
\text { Types } & \tau & ::= & \text { int }_{\ell} \mid \text { int }_{\ell} \text { ref } \mid \text { stmt }_{\mathcal{R}}
\end{array}
$$

In Aimp, the only data type is int $\ell$, an integer type annotated with security label $\ell$, which contains three base labels as described in Section 2.8.

A memory reference $m$ has type int $\ell_{\ell}$ ref, indicating the value stored at $m$ has type $\operatorname{int}_{\ell}$. In Aimp, types of memory references are specified by a typing assignment $\Gamma$ that maps references to types so that the type of $m$ is $\tau$ ref if $\Gamma(m)=\tau$.

The type of a statement $s$ has the form $\operatorname{stmt}_{\mathcal{R}}$ where $\mathcal{R}$ contains the set of unassigned output references when $s$ terminates. Intuitively, $\mathcal{R}$ represents all the outputs that are still expected by users after $s$ terminates.

\subsection{Operational semantics}

The small-step operational semantics of Aimp is given in Figure 2. Let $M$ represent a memory that is a finite map from locations to values (including none), and let $\langle s, M\rangle$ be a machine configuration. Then a small evaluation step is a transition from $\langle s, M\rangle$ to another configuration $\left\langle s^{\prime}, M^{\prime}\right\rangle$, written $\langle s, M\rangle \longmapsto\left\langle s^{\prime}, M^{\prime}\right\rangle$.

The evaluation rules (S1)-(S6) are standard for an imperative language. Rules (E1) and (E2) are used to evaluate expressions. Because an expression has no side-effect, we use the notation $\langle e, M\rangle \Downarrow v$ to mean that evaluating $e$ in memory $M$ results in the value $v$. Rule (E1) is used to evaluate dereference expression $! m$. In rule (E2), $v_{1}+v_{2}$ is computed using the following formula:

$$
v_{1}+v_{2}=\left\{\begin{array}{lll}
n_{1}+n_{2} & \text { if } & v_{1}=n_{1} \text { and } v_{2}=n_{2} \\
\text { none } & \text { if } & v_{1}=\text { none or } v_{2}=\text { none }
\end{array}\right.
$$

Rules (S1), (S4) and (S5) show that if the evaluation of configuration $\langle s, M\rangle$ depends on the result of an expression $e$, it must be the case that $\langle e, M\rangle \Downarrow n$. In other words, if $\langle e, M\rangle \Downarrow$ none, the evaluation of $\langle s, M\rangle$ gets stuck.

\subsection{Examples}

By its simplicity, the Aimp language helps focus on the essentials of an imperative language. Figure 3 shows a few code segments that demonstrate various kind of availability dependencies, some of which are subtle. In all these examples, $m_{o}$ represents an output, and its initial value is none. All other references represent inputs.

In code segment (A), if $m_{1}$ is unavailable, the execution gets stuck at the first assignment. Therefore, the availability of $m_{o}$ depends on the availability of $m_{1}$.

$$
\begin{aligned}
& \text { [E1] } \quad \frac{m \in \operatorname{dom}(M)}{\langle! m, M\rangle \Downarrow M(m)} \\
& \text { [E2] } \frac{\left\langle e_{1}, M\right\rangle \Downarrow v_{1} \quad\left\langle e_{2}, M\right\rangle \Downarrow v_{2} \quad v=v_{1}+v_{2}}{\left\langle e_{1}+e_{2}, M\right\rangle \Downarrow v} \\
& \frac{\langle e, M\rangle \Downarrow n}{\langle m:=e, M\rangle \longmapsto\langle\text { skip, } M[m \longmapsto n]\rangle} \\
& \frac{\left\langle s_{1}, M\right\rangle \longmapsto\left\langle s_{1}^{\prime}, M^{\prime}\right\rangle}{\left\langle s_{1} ; s_{2}, M\right\rangle \longmapsto\left\langle s_{1}^{\prime} ; s_{2}, M^{\prime}\right\rangle} \\
& \langle\text { skip; } s, M\rangle \longmapsto\langle s, M\rangle \\
& \frac{\langle e, M\rangle \Downarrow n \quad n>0}{\left\langle\text { if } e \text { then } s_{1} \text { else } s_{2}, M\right\rangle \longmapsto\left\langle s_{1}, M\right\rangle} \\
& \frac{\langle e, M\rangle \Downarrow n \quad n \leq 0}{\left\langle\text { if } e \text { then } s_{1} \text { else } s_{2}, M\right\rangle \longmapsto\left\langle s_{2}, M\right\rangle} \\
& \langle\text { while } e \text { do } s, M\rangle \longmapsto \\
& \langle\text { if } e \text { then } s \text {; while } e \text { do } s \text { else skip, } M\rangle
\end{aligned}
$$

Figure 2. Operational semantics for Aimp
(A) $m_{2}:=! m_{1} ; \quad m_{o}:=1$;

(B) while $\left(! m_{1}\right)$ do skip; $\quad m_{o}:=1$;

(C) if $\left(! m_{1}\right)$ then while (1) do skip; else skip; $m_{o}:=1$;

(D) if $\left(! m_{1}\right)$ then $m_{o}:=1$ else skip; while $\left(! m_{2}\right)$ do skip; $m_{o}:=2$;

Figure 3. Examples

In code segment (B), the while statement gets stuck if $m_{1}$ is unavailable. Moreover, it diverges if the value of $m_{1}$ is positive. Thus, the availability of $m_{o}$ depends on both the availability and the value of $m_{1}$.

In code segment $(\mathrm{C})$, the if statement does not terminate if $m_{1}$ is positive, so the availability of $m_{o}$ depends on the value of $m_{1}$.

In code segment (D), $m_{o}$ is assigned in one branch of the if statement, but not in the other. Therefore, when the if statement terminates, the availability of $m_{o}$ depends on the value of $m_{1}$. Moreover, the program executes a while statement that may diverge before $m_{o}$ is assigned value 2 . Therefore, for the whole program, the availability of $m_{o}$ depends on the value of $m_{1}$. 


\section{Noninterference properties}

This section formalizes the noninterference properties (in particular, availability noninterference) that correspond to the security policies of Section 2. Although this formalization is done in the context of Aimp, it can be easily generalized to other state transition systems.

For both confidentiality and integrity, noninterference has a simple, intuitive description: equivalent low-confidentiality (high-integrity) inputs always result in equivalent low-confidentiality (high-integrity) outputs. The notion of availability noninterference is more subtle, because an attacker has two ways to compromise the availability of an output. First, the attacker can make an input unavailable and block the computation using the input. Second, the attacker can try to affect the integrity of control flow and make the program diverge (fail to terminate). In other words, the availability of an output may depend on both the integrity and availability of an input. The observation is captured by this intuitive description of availability noninterference:

With all high-availability inputs available, equivalent high-integrity inputs will eventually result in equally available high-availability outputs.

As far as we are aware, no previous work has proposed a notion of noninterference between the availability of outputs and both the integrity and availability of inputs. This formulation of noninterference provides a separation of concerns (and policies) for availability and integrity, yet prevents the two attacks discussed above.

The intuitive concepts of high and low security are based on the power of the potential attacker, which is represented by a base label $L$. In the DLM, suppose the attacker is able to act for principals $p_{1}, \ldots, p_{n}$, and that there exists a top principal (denoted by $*$ ) that acts for every principal. Then we have $L=\left\{*: p_{1} \wedge \ldots \wedge p_{n}\right\}$, because $p_{1} \wedge \ldots \wedge p_{n}$ is the most powerful principal that the attacker controls. Given a base label $\beta$, if $\beta \leq L$ then the label represents a lowsecurity level that is not protected from the attacker. Otherwise, $\beta$ is a high-security label.

For an imperative language, the inputs of a program are just the initial memory, and the outputs are the observable aspects of a program execution, which is defined by the $o b$ servation model of the language. In Aimp, we have the following observation model:

- Memories are observable.

- The value none is not observable. In other words, if $M(m)=$ none, an observer cannot determine the value of $m$ in $M$.

Suppose $s$ is a program, and $M$ is the initial memory. Based on the observation model, the outputs of $s$ are a set $\mathcal{T}$ of finite traces of memories, and for any trace $T$ in $\mathcal{T}$, there exists an evaluation $\langle s, M\rangle \longmapsto\left\langle s_{1}, M_{1}\right\rangle \longmapsto \ldots \longmapsto$ $\left\langle s_{n}, M_{n}\right\rangle$ such that $T=\left[M, M_{1}, \ldots, M_{n}\right]$. Intuitively, every trace in $\mathcal{T}$ is the outputs observable to users at some point during the evaluation of $\langle s, M\rangle$, and $\mathcal{T}$ represents all the outputs of $\langle s, M\rangle$ observable to users. Since the Aimp language is deterministic, for any two traces in $\mathcal{T}$, it must be the case that one is a prefix of the other.

In the intuitive description of noninterference, equivalent low-confidentiality inputs can be represented by two memories whose low-confidentiality parts are indistinguishable. Suppose the typing information of a memory $M$ is given by a typing assignment $\Gamma$. Then $m$ belongs to the low-confidentiality part of $M$ if $C(\Gamma(m)) \leq L$, where $C(\Gamma(m))$ denotes $C(\ell)$ if $\Gamma(m)$ is int $_{\ell}$. Similarly, $m$ is a high-integrity reference if $I(\Gamma(m)) \not \leq L$, and a highavailability reference if $A(\Gamma(m)) \not L L$. Let $v_{1} \approx v_{2}$ denote that $v_{1}$ and $v_{2}$ are indistinguishable. By the observation model of Aimp, a user cannot distinguish none from any other value. Consequently, $v_{1} \approx v_{2}$ if and only if $v_{1}=v_{2}$, $v_{1}=$ none or $v_{2}=$ none. With these settings, given two memories $M_{1}$ and $M_{2}$ with respect to $\Gamma$, we define three kinds of indistinguishability relations between $M_{1}$ and $M_{2}$ as follows:

Definition 4.1 ( $\left.\Gamma \vdash M_{1} \quad \approx_{C<L} \quad M_{2}\right)$. The lowconfidentiality parts of $M_{1}$ and $M_{2}$ are indistinguishable, written $\Gamma \vdash M_{1} \approx_{C \leq L} M_{2}$, if for any $m \in \operatorname{dom}(\Gamma)$, $C(\Gamma(m)) \leq L$ implies $M_{1}(m) \approx M_{2}(m)$.

Definition $4.2\left(\Gamma \vdash M_{1} \approx_{I \not \subset L} M_{2}\right)$. The high-integrity parts of $M_{1}$ and $M_{2}$ are indistinguishable, written $\Gamma \vdash$ $M_{1} \approx_{I \not L L} M_{2}$, if for any $m \in \operatorname{dom}(\Gamma), I(\Gamma(m)) \not \leq L$ implies $M_{1}(m) \approx M_{2}(m)$.

Definition $4.3\left(\Gamma \vdash M_{1} \approx_{A \not L L} M_{2}\right)$. The high-availability parts of $M_{1}$ and $M_{2}$ are equally available, written $\Gamma \vdash$ $M_{1} \approx_{A \not L L} M_{2}$, if for any $m \in \operatorname{dom}(\Gamma), A(\Gamma(m)) \not \leq L$ implies that $M_{1}(m)=$ none if and only if $M_{2}(m)=$ none.

Based on the definitions of memory indistinguishability, we can define trace indistinguishability, which formalizes the notion of equivalent outputs. First, we assume that users cannot observe timing. As a result, traces $[M, M]$ and $[M]$ look the same to a user. In general, two traces $T_{1}$ and $T_{2}$ are equivalent, written $T_{1} \approx T_{2}$, if they are equal up to stuttering, which means the two traces obtained by eliminating repeated elements in $T_{1}$ and $T_{2}$ are equal. For example, $\left[M_{1}, M_{2}, M_{2}\right] \approx\left[M_{1}, M_{1}, M_{2}\right]$. Second, $T_{1}$ and $T_{2}$ are indistinguishable, if $T_{1}$ appears to be a prefix of $T_{2}$, because in that case, $T_{1}$ and $T_{2}$ may be generated by the same execution. Given two traces $T_{1}$ and $T_{2}$ of memories with respect to $\Gamma$, let $\Gamma \vdash T_{1} \approx_{C \leq L} T_{2}$ denote that the lowconfidentiality parts of $T_{1}$ and $T_{2}$ are indistinguishable, and $\Gamma \vdash T_{1} \approx_{I \not \subset L} T_{2}$ denote that the high-integrity parts of $T_{1}$ and $T_{2}$ are indistinguishable. These two notions are defined as follows: 
Definition 4.4 ( $\left.\Gamma \vdash T_{1} \approx_{C \leq L} T_{2}\right)$. Given two traces $T_{1}$ and $T_{2}, \Gamma \vdash T_{1} \approx_{C \leq L} T_{2}$ if there exists $T_{1}^{\prime}=\left[M_{1}, \ldots, M_{n}\right]$ and $T_{2}^{\prime}=\left[M_{1}^{\prime}, \ldots, M_{m}^{\prime}\right]$ such that $T_{1} \approx T_{1}^{\prime}$, and $T_{2} \approx T_{2}^{\prime}$, and $\Gamma \vdash M_{i} \approx_{C \leq L} M_{i}^{\prime}$ for any $i$ in $\{1, \ldots, \min (m, n)\}$.

Definition $4.5\left(\Gamma \vdash T_{1} \approx_{I \not L L} T_{2}\right)$. Given two traces $T_{1}$ and $T_{2}, \Gamma \vdash T_{1} \approx_{I \not L L} T_{2}$ if there exists $T_{1}^{\prime}=\left[M_{1}, \ldots, M_{n}\right]$ and $T_{2}^{\prime}=\left[M_{1}^{\prime}, \ldots, M_{m}^{\prime}\right]$ such that $T_{1} \approx T_{1}^{\prime}$, and $T_{2} \approx T_{2}^{\prime}$, and $\Gamma \vdash M_{i} \approx_{I \not L L} M_{i}^{\prime}$ for any $i$ in $\{1, \ldots, \min (m, n)\}$.

Note that two executions are indistinguishable if any two finite traces generated by those two executions are indistinguishable. Thus, we can still reason about the indistinguishability of two nonterminating executions, even though $\approx_{I \not L L}$ and $\approx_{C \leq L}$ are defined on finite traces.

With the formal definitions of memory indistinguishability and trace indistinguishability, it is straightforward to formalize confidentiality noninterference and integrity noninterference:

Definition 4.6 (Confidentiality noninterference). A program $s$ has the confidentiality noninterference property w.r.t. a typing assignment $\Gamma$, written $\Gamma \vdash \mathrm{NI}_{C}(s)$, if for any two traces $T_{1}$ and $T_{2}$ generated by evaluating $\left\langle s, M_{1}\right\rangle$ and $\left\langle s, M_{2}\right\rangle$, we have that $\Gamma \vdash M_{1} \approx_{C \leq L} M_{2}$ implies $\Gamma \vdash T_{1} \approx_{C \leq L} T_{2}$.

Note that this confidentiality noninterference property does not treat covert channels based on termination and timing. Static control of timing channels is largely orthogonal to this work, and has been partially addressed elsewhere [28, 2, 23].

Definition 4.7 (Integrity noninterference). A program $s$ has the integrity noninterference property w.r.t. a typing assignment $\Gamma$, written $\Gamma \vdash \mathrm{NI}_{I}(s)$, if for any two traces $T_{1}$ and $T_{2}$ generated by evaluating $\left\langle s, M_{1}\right\rangle$ and $\left\langle s, M_{2}\right\rangle$, we have that $\Gamma \vdash M_{1} \approx_{I \not \subset L} M_{2}$ implies $\Gamma \vdash T_{1} \approx_{I \not L L} T_{2}$.

Consider the intuitive description of availability noninterference. To formalize the notion that all the highavailability inputs are available, we first need to distinguish input references from unassigned output references. Given a program $s$, let $\mathcal{R}$ denote the set of unassigned output references. In general, references in $\mathcal{R}$ are mapped to none in the initial memory. If $m \notin \mathcal{R}$, then reference $m$ represents either an input, or an output that is already been generated. Thus, given an initial memory $M$, the notion that all the high-availability inputs are available can be represented by $\forall m .(A(\Gamma(m)) \not L L \wedge m \notin \mathcal{R}) \Rightarrow M(m) \neq$ none, as in the following definition of availability noninterference:

Definition 4.8 (Availability noninterference). A program $s$ has the availability noninterference property w.r.t. a typing assignment $\Gamma$ and a set of unassigned output references $\mathcal{R}$, written $\Gamma ; \mathcal{R} \vdash \mathrm{NI}_{A}(s)$, if for any two memories $M_{1}, M_{2}$, the following statements
- $\Gamma \vdash M_{1} \approx_{I \not \subset L} M_{2}$

- For $i \in\{1,2\}, \forall m \in \operatorname{dom}(\Gamma) . A(\Gamma(m)) \not L L \wedge m \notin$ $\mathcal{R} \Rightarrow M_{i}(m) \neq$ none

- $\left\langle s, M_{i}\right\rangle \longmapsto{ }^{*}\left\langle s_{i}^{\prime}, M_{i}^{\prime}\right\rangle$ for $i \in\{1,2\}$

imply that there exist $\left\langle s_{i}^{\prime \prime}, M_{i}^{\prime \prime}\right\rangle$ for $i \in\{1,2\}$ such that $\left\langle s_{i}^{\prime}, M_{i}^{\prime}\right\rangle \longmapsto^{*}\left\langle s_{i}^{\prime \prime}, M_{i}^{\prime \prime}\right\rangle$ and $\Gamma \vdash M_{1}^{\prime \prime} \approx_{A \not L L} M_{2}^{\prime \prime}$.

\section{Security typing and soundness}

The type system of Aimp is designed to ensure that any well-typed Aimp program satisfies the noninterference properties defined in Section 4. For confidentiality and integrity, the type system performs a standard static information flow analysis [7, 30]. For availability, the type system tracks the set of unassigned output references and uses them to ensure that availability requirements are not violated.

To track unassigned output references, the typing environment for a statement $s$ includes a component $\mathcal{R}$, which contains the set of unassigned output references before the execution of $s$. The typing judgment for statements has the form: $\Gamma ; \mathcal{R} ; p c \vdash s:$ stmt $_{\mathcal{R}^{\prime}}$, where $\Gamma$ is the typing assignment, and $p c$ is the program counter label [6] used to track security levels of the program counter. The typing judgment for expressions has the form $\Gamma ; \mathcal{R} \vdash e: \tau$

The typing rules are shown in Figure 5. Rules (INT) and (NONE) check constants. An integer $n$ has type int $t_{\ell}$ where $\ell$ can be an arbitrary label. The value none represents an unavailable value, so it can have any data type. Since int is the only data type in Aimp, none has type int . $_{\text {. }}$

Rule (REF) says that the type of a reference $m$ is $\tau$ ref if $\Gamma(m)=\tau$. In Aimp, a memory maps references to values, and values always have integer types.

Rule (DEREF) checks dereference expressions. It disallows dereferencing the references in $\mathcal{R}$, because they are unassigned output references.

Rule (ADD) checks addition expressions. Let $\ell_{1} \sqcup \ell_{2}$ be $\left\langle C\left(\ell_{1}\right) \sqcup C\left(\ell_{2}\right), I\left(\ell_{1}\right) \sqcap I\left(\ell_{2}\right), A\left(\ell_{1}\right) \sqcap A\left(\ell_{2}\right)\right\rangle$. As discussed in Section 2.8, the label of $e_{1}+e_{2}$ is exactly $\ell_{1} \sqcup \ell_{2}$ if $e_{i}$ has the label $\ell_{i}$ for $i \in\{1,2\}$.

Rule (SEQ) checks sequential statements. The premise $\Gamma ; \mathcal{R} ; p c \vdash s_{1}: \operatorname{stmt}_{\mathcal{R}_{1}}$ means that $\mathcal{R}_{1}$ is the set of unassigned output references after $s_{1}$ terminates and before $s_{2}$ starts. Therefore, the typing environment for $s_{2}$ is $\Gamma ; \mathcal{R}_{1} ; p c$. It is clear that $s_{2}$ and $s_{1} ; s_{2}$ terminate at the same point. Thus, $s_{1} ; s_{2}$ has the same type as $s_{2}$.

Rule (ASSIGN) checks assignment statements. The statement $m:=e$ assigns the value of $e$ to $m$, creating an explicit information flow from $e$ to $m$ and an implicit flow from the program counter to $m$. To control these information flows, this rule requires $C\left(\ell^{\prime}\right) \sqcup C(p c) \leq C(\Gamma(m))$ to protect the confidentiality of $e$ and the program counter, and $I(\Gamma(m)) \leq I(p c) \sqcap C\left(\ell^{\prime}\right)$ to protect the integrity of $m$. 
$[I N T]$

$$
\Gamma ; \mathcal{R} \vdash n: \operatorname{int}_{\ell}
$$

$[N O N E]$

$$
\Gamma ; \mathcal{R} \vdash \text { none }^{\text {int }_{\ell}}
$$

$[R E F]$

[DEREF]

$$
\begin{gathered}
\frac{m \notin \mathcal{R} \quad \Gamma(m)=\text { int }_{\ell}}{\Gamma ; \mathcal{R} \vdash ! m: \text { int }_{\ell}} \\
\frac{\Gamma ; \mathcal{R} \vdash e_{1}: \text { int }_{\ell_{1}} \quad \Gamma ; \mathcal{R} \vdash e_{2}: \text { int }_{\ell_{2}}}{\Gamma ; \mathcal{R} \vdash e_{1}+e_{2}: \text { int }_{\ell_{1}} \sqcup \ell_{2}} \\
\Gamma ; \mathcal{R} ; p c \vdash \text { skip }: \text { stmt }_{\mathcal{R}} \\
\frac{\Gamma ; \mathcal{R} ; p c \vdash s_{1}: \text { stmt }_{\mathcal{R}_{1}}}{\Gamma ; \mathcal{R}{ }_{1} ; p c \vdash s_{2}: \text { stmt }_{\mathcal{R}_{2}}} \\
\Gamma ; p c \vdash s_{1} ; s_{2}: \text { stmt }_{\mathcal{R}_{2}}
\end{gathered}
$$

[ADD]

[SKIP]

[SEQ]

[ASSIGN]

$$
\Gamma ; \mathcal{R} \vdash m \text { int }_{\ell} \text { ref } \quad \Gamma ; \mathcal{R} \vdash e: \text { int }_{\ell^{\prime}}
$$$$
C(p c) \sqcup C\left(\ell^{\prime}\right) \leq C(\ell) \quad I(\ell) \leq I(p c) \sqcap I\left(\ell^{\prime}\right)
$$

$$
\bar{A}_{\Gamma}(\mathcal{R}) \leq A\left(\ell^{\prime}\right)
$$$$
\Gamma ; \mathcal{R} ; p c \vdash m:=e: \operatorname{stmt}_{\mathcal{R}-\{m\}}
$$$$
\Gamma ; \mathcal{R} \vdash e: \operatorname{int}_{\ell} \quad A_{\Gamma}(\mathcal{R}) \leq A(\ell)
$$

[WHILE]

$$
\Gamma ; \mathcal{R} ; p c \sqcup \ell \vdash s_{i}: \tau \quad i \in\{1,2\}
$$

$\Gamma ; \mathcal{R} ; p c \vdash$ if $e$ then $s_{1}$ else $s_{2}: \tau$

$$
\begin{gathered}
\Gamma \vdash e: \text { int }_{\ell} \quad \Gamma ; \mathcal{R} ; p c \sqcup \ell \vdash s: \text { stmt }_{\mathcal{R}} \\
A_{\Gamma}(\mathcal{R}) \leq I(\ell) \sqcap I(p c) \sqcap A(\ell) \\
\Gamma ; \mathcal{R} ; p c \vdash \text { while } e \text { do } s: \text { stmt }_{\mathcal{R}}
\end{gathered}
$$

$$
\frac{\Gamma ; \mathcal{R} ; p c \vdash s: \tau \quad \Gamma ; \mathcal{R} ; p c \vdash \tau \leq \tau^{\prime}}{\Gamma ; \mathcal{R} ; p c \vdash s: \tau^{\prime}}
$$

Figure 4. Typing rules for Aimp

If the value of $e$ is unavailable, the assignment $m:=e$ will get stuck. Therefore, rule (ASSIGN) has the premise $A_{\Gamma}(\mathcal{R}) \leq A\left(\ell^{\prime}\right)$, where $A_{\Gamma}(\mathcal{R})=\bigsqcup_{m \in \mathcal{R}} A(\Gamma(m))$, to ensure the availability of $e$ is as high as the availability of any unassigned output reference. For example, in the code segment (A) of Figure 3, the type system ensures that $A\left(\Gamma\left(m_{o}\right)\right) \leq A\left(\Gamma\left(m_{1}\right)\right)$.

Finally, when the assignment $m:=e$ terminates, $m$ should be removed from the set of unassigned output references, and thus the statement has type $\operatorname{stmt}_{\mathcal{R}-\{m\}}$.

Rule (IF) checks if statements. Consider the statement if $e$ then $s_{1}$ else $s_{2}$. The value of $e$ determines which branch is executed, so the program-counter labels for branches $s_{1}$ and $s_{2}$ subsume the label of $e$ to protect $e$ from implicit flows. As usual, the if statement has type $\tau$ if both $s_{1}$ and $s_{2}$ have type $\tau$. As in rule (AS-
SIGN), the premise $A_{\Gamma}(R) \leq A(\ell)$ ensures that $e$ has sufficient availability.

Rule (WHILE) checks while statements. In this rule, the premise $A_{\Gamma}(\mathcal{R}) \leq I(\ell) \sqcap I(p c) \sqcap A(\ell)$ can be decomposed into three constraints: $A_{\Gamma}(\mathcal{R}) \leq A(\ell)$, which ensures that $e$ has sufficient availability, $A_{\Gamma}(\mathcal{R}) \leq I(\ell)$, which prevents attackers from making the while statement diverge by compromising the integrity of $e$, and $A_{\Gamma}(\mathcal{R}) \leq I(p c)$, which guarantees the integrity of the control flow reaching the while statement, because a while statement may diverge without any interaction with attackers.

For example, consider the code segments (B) and (C) in Figure 3, in which $\mathcal{R}=\left\{m_{o}\right\}$. Suppose $A\left(\Gamma\left(m_{o}\right)\right) \not L L$. In (B), the constraint $A_{\Gamma}(\mathcal{R}) \leq I(\ell)$ of rule (WHILE) ensures $I\left(\Gamma\left(m_{1}\right)\right) \not \leq L$, so attackers cannot affect the value of $m_{1}$, and whether the while statement diverges. In (C), the constraint $A_{\Gamma}(\mathcal{R}) \leq I(p c)$ guarantees $I(p c) \not \leq L$, and thus $I\left(\Gamma\left(m_{1}\right)\right) \not \leq L$ holds because $I(p c) \leq I\left(\Gamma\left(m_{1}\right)\right)$. Therefore, attackers cannot affect which branch of the if statement would be taken, or whether control reaches the while statement.

Rule (SUB) is the standard subsumption rule. Let $\Gamma ; \mathcal{R} ; p c \vdash \tau \leq \tau^{\prime}$ denote that $\tau$ is a subtype of $\tau^{\prime}$ with respect to the typing environment $\Gamma ; \mathcal{R} ; p c$. The type system of Aimp has one subtyping rule:

$$
\begin{gathered}
\mathcal{R}^{\prime} \subseteq \mathcal{R}^{\prime \prime} \subseteq \mathcal{R} \\
\forall m, m \in \mathcal{R}^{\prime \prime}-\mathcal{R}^{\prime} \Rightarrow A(\Gamma(m)) \leq I(p c) \\
\Gamma ; \mathcal{R} ; p c \vdash \text { stmt }_{\mathcal{R}^{\prime}} \leq \text { stmt }_{\mathcal{R}^{\prime \prime}}
\end{gathered}
$$

Suppose $\Gamma ; \mathcal{R} ; p c \vdash \operatorname{stmt}_{\mathcal{R}^{\prime}} \leq \operatorname{stmt}_{\mathcal{R}^{\prime \prime}}$ and $\Gamma ; \mathcal{R} ; p c \vdash$ $s: \operatorname{stmt}_{\mathcal{R}^{\prime}}$. Then $\Gamma ; \mathcal{R} ; p c \vdash s: \operatorname{stmt}_{\mathcal{R}^{\prime \prime}}$ by rule (SUB). In other words, if $\mathcal{R}^{\prime}$ contains all the unassigned output references after $s$ terminates, so does $\mathcal{R}^{\prime \prime}$. This is guaranteed by the premise $\mathcal{R}^{\prime} \subseteq \mathcal{R}^{\prime \prime}$ of rule (ST). The reference set $\mathcal{R}$ contains all the unassigned output references before $s$ is executed, so rule (ST) requires $\mathcal{R}^{\prime \prime} \subseteq \mathcal{R}$. Intuitively, that the statement $s$ can be treated as having type stmt $\mathcal{R}^{\prime \prime}$ is because there exists another control flow path that bypasses $s$ and does not assign to references in $\mathcal{R}^{\prime \prime}-\mathcal{R}^{\prime}$. Consequently, for any $m$ in $\mathcal{R}^{\prime \prime}-\mathcal{R}^{\prime}$, the availability of $m$ may depend on whether $s$ is executed. Therefore, rule (ST) enforces the constraint $\forall m, m \in \mathcal{R}^{\prime \prime}-\mathcal{R}^{\prime} \Rightarrow A(\Gamma(m)) \leq I(p c)$.

Consider the assignment $m_{o}:=1$ in code segment (D) of Figure 3. By rule (ASSIGN), $\Gamma ;\left\{m_{o}\right\} ; p c \vdash m_{o}:=$ 0 : stmt $_{\emptyset}$. For the else branch of the if statement, we have $\Gamma ;\left\{m_{o}\right\} ; p c \vdash$ skip : stmt $\left\{m_{o}\right\}$. By rule (IF), $\Gamma ;\left\{m_{o}\right\} ; p c \vdash m_{o}:=0: \operatorname{stmt}_{\left\{m_{o}\right\}}$ needs to hold, which requires $\Gamma ;\left\{m_{o}\right\} ; p c \vdash \operatorname{stmt}_{\emptyset} \leq \operatorname{stmt}_{\left\{m_{o}\right\}}$. In this example, the availability of $m_{o}$ depends on which branch is taken, and we need to ensure $A\left(\Gamma\left(m_{o}\right)\right) \leq I\left(\Gamma\left(m_{1}\right)\right)$. Indeed, if (D) is well typed, by rules (ST) and (IF), we have $A\left(\Gamma\left(m_{o}\right)\right) \leq I(p c) \leq I\left(\Gamma\left(m_{1}\right)\right)$. 
This type system satisfies the subject reduction property. Moreover, we can prove that any well-typed program has confidentiality, integrity and availability noninterference properties. These results are formalized in the following two theorems (see the technical report [34] for the proofs).

Theorem 5.1 (Subject reduction). Suppose $\Gamma ; \mathcal{R} ; p c \vdash$ $s: \tau$, and $\operatorname{dom}(\Gamma)=\operatorname{dom}(M)$. If $\langle s, M\rangle \longmapsto\left\langle s^{\prime}, M^{\prime}\right\rangle$, then there exists $\mathcal{R}^{\prime}$ such that $\Gamma ; \mathcal{R}^{\prime} ; p c \vdash s^{\prime}: \tau$, and $\mathcal{R}^{\prime} \subseteq \mathcal{R}$, and for any $m \in \mathcal{R}-\mathcal{R}^{\prime}, M^{\prime}(m) \neq$ none.

Theorem 5.2 (Noninterference). If $\Gamma ; \mathcal{R} ; p c \vdash s: \tau$, then $\Gamma \vdash \mathrm{NI}_{C}(s), \Gamma \vdash \mathrm{NI}_{I}(s)$ and $\Gamma ; \mathcal{R} \vdash \mathrm{NI}_{A}(s)$.

\section{Extensions}

This section describes two language extensions that can be used to reduce availability dependencies and allow a program to use low-availability data in a more flexible and practical way.

\subsection{Timeout}

Timeouts can effectively turn a blocking operation into a non-blocking operation, and thus provide a strong availability guarantee for a computation that uses low-availability inputs. To support timeouts, we introduce two syntax extensions to Aimp: timed integer values and a race expression.

$$
\begin{array}{rlll|l}
\text { Values } & v & ::= & \ldots & \langle n, t\rangle \\
\text { Expressions } & e & ::= & \ldots & e_{1} \# e_{2}
\end{array}
$$

A timed integer $\langle n, t\rangle$ is similar to integer $n$ except that it would take $t$ units of time to use this value. A race expression $e_{1} \# e_{2}$ evaluates $e_{1}$ and $e_{2}$ at the same time and returns the result of the expression that finishes first. If both $e_{1}$ and $e_{2}$ finish at the same time, the result of $e_{1}$ would be the final result. Suppose we want to set a timeout $t$ for expression $e$ so that if the evaluation of $e$ does not finish in $t$ units of time, a default value $n$ is returned as the result of $e$. This can be implemented by the expression $e \#\langle n, t\rangle$.

Using the timeout mechanism, the following program implements an auction for two clients Alice and Bob. Reference $m_{A}$ represents Alice's bid, and Alice has 30 units of time to make a bid, otherwise time runs out, and 0 is returned as her bid. Similarly, Bob also has 30 units of time to make a bid. Even though the result of this auction depends on the bids of Alice and Bob, the availability of the auction result is not affected by them.

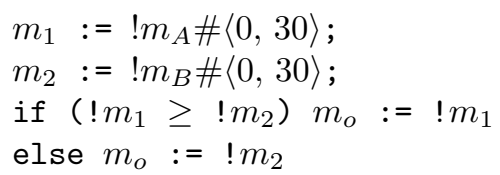

6.1.1. Operational semantics. Note that value $n$ can be treated as a syntax sugar for $\langle n, 0\rangle$. As a result, the evaluation rules in Figure 2 can be adapted to the timeout extension by replacing any occurrence of $\langle e, M\rangle \Downarrow n$ with a more general form $\langle e, M\rangle \Downarrow\langle n, t\rangle$. For example, the adapted rule $(\mathrm{S} 1)$ is shown below:

$$
[S 1] \quad \frac{\langle e, M\rangle \Downarrow\langle n, t\rangle}{\langle m:=e, M\rangle \longmapsto\langle\text { skip, } M[m \longmapsto n]\rangle}
$$

In addition, the formula for computing $v_{1}+v_{2}$ in rule (E2) also needs to be adapted to this more general form of values:

$v_{1}+v_{2}= \begin{cases}\left\langle n_{1}+n_{2}, t_{1}+t_{2}\right\rangle & \text { if } \forall i \in\{1,2\} \cdot v_{i}=\left\langle n_{i}, t_{i}\right\rangle \\ \text { none } & \text { if } v_{1}=\text { none or } v_{2}=\text { none }\end{cases}$

The operational semantics of the race expression is given by the following rules (E3)-(E5). Suppose $e_{1}$ and $e_{2}$ are evaluated to $\left\langle n_{1}, t_{1}\right\rangle$ and $\left\langle n_{2}, t_{2}\right\rangle$, which means evaluating $e_{1}$ and $e_{2}$ takes $t_{1}$ and $t_{2}$ units of time, respectively. Thus, if $t_{1} \leq t_{2}$ (E3), the result of $e_{1}$ should be the final result, and if $t_{1}>t_{2}$ (E4), $\left\langle n_{2}, t_{2}\right\rangle$ is the final result. Rule (E5) applies when only the result of one expression $e_{i}$ is available.

$$
\begin{aligned}
& \text { [E3] } \quad \frac{\left\langle e_{1}, M\right\rangle \Downarrow\left\langle n_{1}, t_{1}\right\rangle \quad\left\langle e_{2}, M\right\rangle \Downarrow\left\langle n_{2}, t_{2}\right\rangle \quad t_{1} \leq t_{2}}{\left\langle e_{1} \# e_{2}, M\right\rangle \Downarrow\left\langle n_{1}, t_{1}\right\rangle} \\
& \text { [E4] } \frac{\left\langle e_{1}, M\right\rangle \Downarrow\left\langle n_{1}, t_{1}\right\rangle \quad\left\langle e_{2}, M\right\rangle \Downarrow\left\langle n_{2}, t_{2}\right\rangle \quad t_{1}>t_{2}}{\left\langle e_{1} \# e_{2}, M\right\rangle \Downarrow\left\langle n_{2}, t_{2}\right\rangle} \\
& \text { [E5] } \frac{\left\langle e_{i}, M\right\rangle \Downarrow\langle n, t\rangle \quad\left\langle e_{j}, M\right\rangle \Downarrow \text { none } \quad\{i, j\}=\{1,2\}}{\left\langle e_{1} \# e_{2}, M\right\rangle \Downarrow\langle n, t\rangle}
\end{aligned}
$$

6.1.2. Typing. The race expression is essential for the timeout mechanism to provide strong availability guarantees. Consider a race expression $e_{1} \# e_{2}$. According to rule (E5), the result of expression $e_{1} \# e_{2}$ is available as long as the result of $e_{1}$ or $e_{2}$ is available. Therefore, the availability of $e$ is as high as the availability of $e_{1}$ and $e_{2}$. Let $A(e)$ represent the availability label of $e$. Then we have $A\left(e_{1} \# e_{2}\right)=A\left(e_{1}\right) \sqcup A\left(e_{2}\right)$. On the other hand, the value of $e_{1} \# e_{2}$ depends on the availability and timing of both $e_{1}$ and $e_{2}$. Consequently, an attacker can try to compromise the integrity of $e_{1} \# e_{2}$ by compromising the availability or timing of $e_{1}$ or $e_{2}$. Intuitively, the race expression trades integrity for availability.

To take into account attacks on timing, a security label may contain a new base label component $\beta_{I T}$ (IT stands for integrity of timing), and $I T(\ell)$ is used to retrieve the component in $\ell$. Suppose expression $e$ has a label $\ell$, and the result of $e$ is $\langle n, t\rangle$. Then an attacker with a security level $L$ can affect the value of $t$ if and only if $I T(\ell) \leq L$.

Suppose $e_{1}$ and $e_{2}$ have type int $\ell_{\ell_{1}}$ and int $_{\ell_{2}}$, respectively. Then $e_{1} \# e_{2}$ has type int $_{\ell_{1} \# \ell_{2}}$, where $\ell_{1} \# \ell_{2}$ is a label computed from $\ell_{1}$ and $\ell_{2}$. Based on the above discussion, we have the following: 
$A\left(\ell_{1} \# \ell_{2}\right)=A\left(\ell_{1}\right) \sqcup A\left(\ell_{2}\right)$

$I\left(\ell_{1} \# \ell_{2}\right)=I\left(\ell_{1}\right) \sqcap I\left(\ell_{2}\right) \sqcap A\left(\ell_{1}\right) \sqcap A\left(\ell_{2}\right) \sqcap I T\left(\ell_{1}\right) \sqcap I T\left(\ell_{2}\right)$

By rule (E5), if the result of $e_{1} \# e_{2}$ is $\langle n, t\rangle$, the value of $t$ may be affected by the availability of $e_{1}$ and $e_{2}$. Therefore,

$$
I T\left(\ell_{1} \# \ell_{2}\right)=I T\left(\ell_{1}\right) \sqcap I T\left(\ell_{2}\right) \sqcap A\left(\ell_{1}\right) \sqcap A\left(\ell_{2}\right)
$$

As usual, $C\left(\ell_{1} \# \ell_{2}\right)=C\left(\ell_{1}\right) \sqcup C\left(\ell_{2}\right)$, since the result of $e_{1} \# e_{2}$ depends on the results of both $e_{1}$ and $e_{2}$. With these formulas for computing $\ell_{1} \# \ell_{2}$, the typing rule for checking the race expression is straightforward:

$$
[R A C E] \frac{\Gamma ; \mathcal{R} \vdash e_{1}: \text { int }_{\ell_{1}} \quad \Gamma ; \mathcal{R} \vdash e_{2}: \text { int }_{\ell_{2}}}{\Gamma ; \mathcal{R} \vdash e_{1} \# e_{2}: \text { int }_{\ell_{1} \# \ell_{2}}}
$$

Because the timeout mechanism trades integrity for availability and allows attackers to compromise the integrity of an output by affecting the availability or timing of an input, the definition of integrity noninterference needs to be adapted to these new risks. Intuitively, the adapted integrity noninterference would require two sets of inputs $M_{1}$ and $M_{2}$ to generate equivalent high-integrity outputs, if the high-integrity parts, the availability of the high-availability parts and the timing of the high-integrity-of-timing parts of $M_{1}$ and $M_{2}$ are indistinguishable. The formal definition is given below, following the definition of the memory indistinguishability with respect to the integrity of timing:

Definition $6.1\left(\Gamma \vdash M_{1} \approx_{I T \not L L} M_{2}\right)$. Suppose $\operatorname{dom}(\Gamma)=$ $\operatorname{dom}\left(M_{1}\right)=\operatorname{dom}\left(M_{2}\right)$. Then $\Gamma \vdash M_{1} \approx_{I T \not \subset L} M_{2}$ means that for any $m \in \operatorname{dom}(\Gamma), I T(\Gamma(m)) \not \leq L$ and $M_{1}(m)=$ $\left\langle n_{1}, t_{1}\right\rangle$ and $M_{2}(m)=\left\langle n_{1}, t_{2}\right\rangle$ imply $t_{1}=t_{2}$.

Definition 6.2 (Integrity noninterference). A program $s$ has the integrity noninterference property w.r.t. a typing assignment $\Gamma$, written $\Gamma \vdash \mathrm{NI}_{I}(s)$, if for any two traces $T_{1}$ and $T_{2}$ generated by evaluating $\left\langle s, M_{1}\right\rangle$ and $\left\langle s, M_{2}\right\rangle$, we have that $\Gamma \vdash M_{1} \approx_{I \not \subset L} \quad M_{2}, \Gamma \vdash M_{1} \approx_{A \not L L} \quad M_{2}$ and $\Gamma \vdash M_{1} \approx_{I T \not \subset L} M_{2}$ imply $\Gamma \vdash T_{1} \approx_{I \not \subset L} T_{2}$.

\subsection{Run-time reference generation}

For a program $s$ in Aimp, the set of outputs that $s$ is expected to generate are statically determined by a set of references $\mathcal{R}$. However, in some realistic applications, an output may be expected only after control reaches certain program points. For example, consider a simple service that responds to the request from a client. The response is expected only after the service receives a client request. To express such kind of availability requirements, we extend Aimp with a new statement that creates a new reference in memory. Intuitively, the output represented by this reference is expected by users only after the point where it is created. The syn- tax of this extension is shown below:

$$
\begin{array}{rlll}
\text { References } & r & := & m \mid x \\
\text { Expressions } & e & := & \ldots \mid ! \\
\text { Statements } & s & := & \ldots \mid r:=e \\
& & \text { new } x: \ell_{x}=\operatorname{ref}(\ell) \text { in } s
\end{array}
$$

The name $x$ is used to range over a set of reference variables. The new statement new $x: \ell_{x}=\operatorname{ref}(\ell)$ in $s$ creates a new reference $m$ with type int $_{\ell}$ ref, substitutes the occurrences of $x$ in $s$ with $m$, and then executes $s$. Now a reference $r$ may be a memory location $m$ or a variable $x$. Accordingly, the dereference expression and the assignment statement have the form $! r$ and $r:=e$, respectively.

Because the memory is observable to users, the creation of a new reference is an observable event and may be used as an information channel. In a new statement new $x: \ell_{x}=$ ref $(\ell)$ in $s$, the label $\ell_{x}$ is used to specify the security level of this event and control this new kind of implicit flows. For example, any user with a confidentiality level not as high as $C\left(\ell_{x}\right)$ should not observe the creation of the reference.

Consider the simple service example. In Aimp, a straightforward implementation is shown below:

$$
\begin{aligned}
& m:=! m_{1} ; \\
& m_{2}:=1 ;
\end{aligned}
$$

where $m_{1}$ represents the client request, and $m_{2}$ represents the output generated by the server in response to the client request. This implementation is problematic because the availability of $m_{2}$ depends on that of $m_{1}$. In practice, we can imagine that the availability labels of $m_{1}$ and $m_{2}$ are $\{*: c l i e n t\}$ and $\{*:$ server $\}$, respectively, where client represents the client machine, and server represents the server machine. However, in general, client does not act for server, and thus $\{*$ : server $\} \not\{\{*$ : client $\}$. Therefore, the above program is not well-typed in practice.

With the new statement, the simple service can be implemented by the following program in which the server response is represented by a reference variable $x$ instead of a memory location. Since $x$ is created after $m_{1}$ is dereferenced, the availability of $x$ does not depend on that of $m_{1}$.

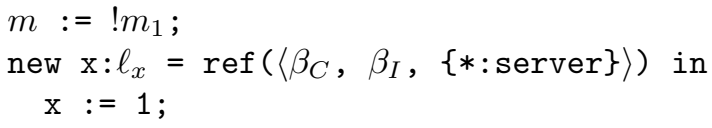

6.2.1. Operational semantics. Formally, the following rule is used to evaluate the new statement:

$$
\begin{gathered}
\frac{m=\operatorname{newloc}\left(M, \ell_{x}\right)}{\left\langle\text { new } x: \ell_{x}=\operatorname{ref}(\ell) \text { in } s, M\right\rangle \longmapsto} \\
\langle s[m / x], M[m \mapsto \text { none }]\rangle
\end{gathered}
$$

The function newloc $\left(M, \ell_{x}\right)$ deterministically returns a fresh reference $m$ such that $m \notin \operatorname{dom}(M)$. The observability and integrity of the newly created reference are specified 
by a label $\ell_{x}$. To associate a memory reference with its label, we assume there exists a map $\Omega$ from the memory space $\mathcal{M}$ (an infinite set of memory locations) to labels. Given a label $\ell$, let $\mathcal{M}_{\ell}=\{m \mid m \in \mathcal{M} \wedge \Omega(m)=\ell\}$. In addition, we assume that for any $\ell, \mathcal{M}_{\ell}$ is infinite. The function newloc $\left(M, \ell_{x}\right)$ deterministically picks a reference $m$ from $\mathcal{M}_{\ell_{x}}$ such that $m \notin \operatorname{dom}(M)$.

The definitions of memory indistinguishability need to take into account the reference labels, which determine the observability and integrity of references themselves. We give the new definition for $\Gamma \vdash M_{1} \approx_{A \not \subset L} M_{2}$ below. Compared to Definition 4.3, this definition does not require $\operatorname{dom}\left(M_{1}\right)=\operatorname{dom}\left(M_{2}\right)$, but $I(\Omega(m)) \not \leq L$ implies $m \in \operatorname{dom}\left(M_{1}\right) \cap \operatorname{dom}\left(M_{2}\right)$. The new definitions for $\Gamma \vdash M_{1} \approx_{I \not L L} \quad M_{2}$ and $\Gamma \vdash M_{1} \approx_{C \leq L} \quad M_{2}$ have similar adjustments.

Definition 6.3 $\left(\Gamma \vdash M_{1} \approx_{A \not \subset L} M_{2}\right)$. Suppose $\operatorname{dom}(\Gamma)=$ $\operatorname{dom}\left(M_{1}\right) \cup \operatorname{dom}\left(M_{2}\right)$. Then $\Gamma \vdash M_{1} \approx_{A \not L L} \quad M_{2}$ if for any $m \in \operatorname{dom}(\Gamma)$ such that $I(\Omega(m)) \not L L$, we have $m \in \operatorname{dom}\left(M_{1}\right) \cap \operatorname{dom}\left(M_{2}\right)$, and $A(\Gamma(m)) \not \leq L$ implies that $M_{1}(m)=$ none if and only if $M_{2}(m)=$ none.

Note that we assume that for any reference $m$ in the initial memory of a program, $\Omega(m)=\left\langle\perp_{C}, \top_{I}, \top_{A}\right\rangle$. As a result, if a program $s$ does not contain any new statement, these new definitions of memory indistinguishability, when applied to the traces of $s$, are consistent with those original definitions in Section 3.

6.2.2. Typing. The type system of Aimp needs to be extended to manipulate reference variables and check the new statement. First, variable $x$ represents a reference that can be used in the typing environment: the typing assignment $\Gamma$ may map $x$ to a type, and the reference set $\mathcal{R}$ may contain $x$. For example, consider the statement new $x: \ell_{x}=$ $\operatorname{ref}(\ell)$ in $s$. Suppose the typing environment for the new statement is " $\Gamma ; \mathcal{R} ; p c$ ". Then the typing environment for $s$ should be " $\Gamma, x:$ int $_{\ell} ; \mathcal{R} \cup\{x\} ; p c$ ". Second, to control the implicit information flow arising from the creation of a new reference, the typing rule for checking the statement new $x: \ell_{x}=\operatorname{ref}(\ell)$ in $s$ needs to ensure that the confidentiality and integrity components of $\ell_{x}$ are bounded by the current program counter label $p c$. Formally, the corresponding constraints are $C(p c) \leq C\left(\ell_{x}\right)$ and $I\left(\ell_{x}\right) \leq I(p c)$.

Intuitively, the value or availability of a reference created at a program point is not affected by whether control reaches this point, because the reference itself does not exist if control does not reach the point. As a result, the typing rules in Figure 5 may be over-restrictive for reasoning about the security policies of a reference created at run time. For example, consider the following code:

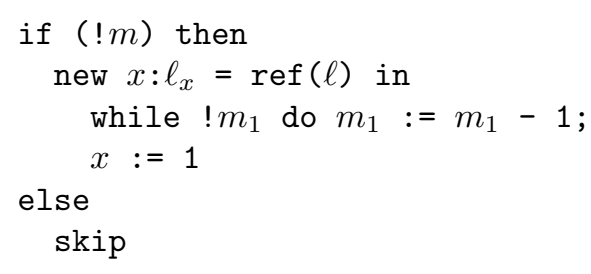

Suppose $\Gamma ; \mathcal{R} ; p c$ is the typing environment for the while statement in the above code. Then $I(p c) \leq I(\Gamma(m))$ holds by the typing rule (IF). Furthermore, we have $x \in \mathcal{R}$, which requires $A(\ell) \leq I(p c)$ by rule (WHILE). Therefore, for the above code to be well-typed, $A(\ell) \leq I(\Gamma(m))$ needs to hold, which contradicts the intuition that the availability of $x$ is not affected by whether control reaches the new statement. To increase the precision of the static security analysis, we extend the type system to track the program counter label for each reference variable $x$ from the program point where $x$ is created. Accordingly, the typing environment is extended with a new component $\Delta$ that maps references to program count labels.

The typing rule (NEW) is used to check the new statement new $x: \ell_{x}=\operatorname{ref}(\ell)$ in $s$. In this rule, statement $s$ is checked with variable $x$ in scope. In the typing environment of $s$, the program counter label mapped to $x$ is $\perp_{p c}$, which is $\left\{\perp_{C}, \top_{I}, \top_{A}\right\}$.

$[N E W]$

$$
\begin{gathered}
\Gamma, x: \text { int }_{\ell} ; \mathcal{R} \cup\{x\} ; \Delta, x: \perp_{p c} ; p c \vdash s: \tau \\
C(p c) \leq C\left(\ell_{x}\right) \quad I\left(\ell_{x}\right) \leq I(p c) \\
\Gamma ; \mathcal{R} ; \Delta ; p c \vdash \text { new } x: \ell_{x}=\operatorname{ref}(\ell) \text { in } s: \tau
\end{gathered}
$$

In addition, typing rules (ASSIGN), (IF) and (WHILE) need to take into account the $\Delta$ component in the typing environment. To abuse the notation a bit, we use $\Delta \sqcup \ell$ to denote the program counter map $\Delta^{\prime}$ that satisfies $\operatorname{dom}(\Delta)=$ $\operatorname{dom}\left(\Delta^{\prime}\right)$ and $\Delta^{\prime}(r)=\Delta(r) \sqcup \ell$ for any $r \in \operatorname{dom}(\Delta)$. In ad-

\begin{tabular}{|c|c|}
\hline \multirow{2}{*}[ASSIGN]{} & $\begin{array}{c}\Gamma ; \mathcal{R} \vdash r: \text { int }_{\ell} \text { ref } \quad \Gamma ; \mathcal{R} \vdash e \text { : }^{\prime} \operatorname{int}_{\ell^{\prime}} \\
C(\Delta(r, p c)) \sqcup C\left(\ell^{\prime}\right) \leq C(\ell) \\
I(\ell) \leq I(\Delta(r, p c)) \sqcap I\left(\ell^{\prime}\right) \quad A_{\Gamma}(\mathcal{R}) \leq A\left(\ell^{\prime}\right.\end{array}$ \\
\hline & $\Gamma ; \mathcal{R} ; \Delta ; p c \vdash r:=e: \operatorname{stmt}_{\mathcal{R}-\{r\}}$ \\
\hline \multirow{2}{*}[IF]{} & $\begin{array}{cc}\Gamma ; \mathcal{R} \vdash e: \operatorname{int}_{\ell} & A_{\Gamma}(\mathcal{R}) \leq A(\ell) \\
\Gamma ; \mathcal{R} ; \Delta \sqcup \ell ; p c \sqcup \ell \vdash s_{i}: \tau \quad i \in\{1,2\}\end{array}$ \\
\hline & $\Gamma ; \mathcal{R} ; \Delta ; p c \vdash$ if $e$ then $s_{1}$ else $s_{2}: \tau$ \\
\hline \multirow{2}{*}{ [WHILE] } & $\begin{array}{c}\Gamma \vdash e: \text { int }_{\ell} \quad A_{\Gamma}(\mathcal{R}) \leq I(\ell) \sqcap A(\ell) \\
\Gamma ; \mathcal{R} ; \Delta \sqcup \ell ; p c \sqcup \ell \vdash s: \operatorname{stmt}_{\mathcal{R}} \\
\forall r \in \mathcal{R}, A(r) \leq I(\Delta(r, p c))\end{array}$ \\
\hline & $\Gamma ; \mathcal{R} ; \Delta ; p c \vdash$ while $e$ do $s:$ stmt $_{\mathcal{R}}$ \\
\hline
\end{tabular}
dition, let $\Delta(r, p c)$ denote $\Delta(r)$ if $r \in \operatorname{dom}(\Delta)$, and $p c$ if otherwise. The adjusted typing rules are shown as follows:

6.2.3. Example: TCP handshake protocol. The TCP connection establishment process uses a three-step handshake protocol [26]. First, a client host $h_{c}$ sends a $\operatorname{SYN}(h)$ 
packet that contains the address of host $h$ to a server $s$. Second, the server sends a SYN_ACK packet to host $h$. Third, host $h$ sends an ACK or RST packet to the server, depending on whether $h$ is $h_{c}$. An instance of this protocol can be simulated by the following code, in which message communications are modeled by assignments:

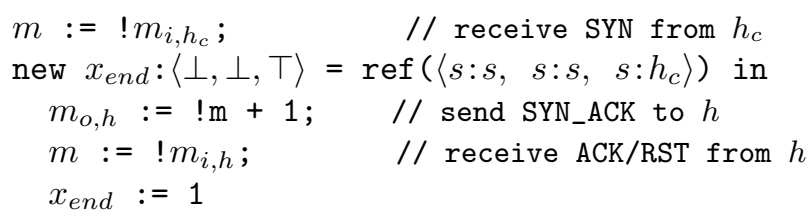

The reference $m_{i, h_{c}}$ represents the connection request from $h_{c}$. After the request is received, a new reference $x_{\text {end }}$ is created to capture the availability requirement of the server: the protocol will terminate if the client $h_{c}$ does not fail, which is specified by the availability label $s: h_{c}$ of $x_{e n d}$.

The statement $m:=m_{i, h}$ represents the third step of the handshake protocol, and the reference $m_{i, h}$ represents the response from $h$. Intuitively, the availability of $m_{i, h}$ only depends on $h$, and thus we suppose that the availability label of $m_{i, h}$ is $s: h$. Then the above code is not well-typed because $s: h_{c} \not \leq s: h$. Interestingly, this reflects the problem with the handshake protocol that allows the SYN flooding attack: host $h$ may be spoofed and cannot be trusted to establish the connection between $s$ and $h_{c}$.

\section{Related work}

There has been much research on ensuring high availability of a computer platform, or guaranteeing a server to carry out the computation requests from clients. Most of these work falls in two main categories: one is aimed at tolerating server-side failures, usually by using some replication techniques $[25,17,4]$; the other deals with faulty clients and defends denial of service attacks [31, 19, 13]. This work is concerned with the availability risks inherent to the computation that may process untrusted inputs, while the computation platform is assumed available.

Lamport first introduced the concepts of safety and liveness properties [15]. Being available is often characterized as a liveness property, which informally means "something good will eventually happen". In general, verifying whether a program will eventually produce an output is equivalent to solving the halting problem, and thus incomputable for a Turing-complete language. In this work, we propose a security model in which an availability policy can be enforced by a noninterference property [9]. It is well known that a noninterference property is not a property on traces [18], and unlike safety or liveness properties, cannot be specified by a trace set. However, a noninterference property can be treated as a property on pairs of traces. For example, consider a trace pair $\left\langle T_{1}, T_{2}\right\rangle$. It has the confidentiality nonin- terference property if the first elements of $T_{1}$ and $T_{2}$ are distinguishable, or $T_{1}$ and $T_{2}$ are indistinguishable to lowconfidentiality users. Therefore, a noninterference property can be represented by a set of trace pairs $S$, and a program satisfies the property if all the pairs of traces produced by the program belong to $S$. Interestingly, with respect to a trace pair, the confidentiality and integrity noninterference properties have the informal meaning of safety properties ("something bad will not happen"), and availability noninterference takes on the informal meaning of liveness.

Focardi and Gorrieri [8] provide a classification of security properties in the setting of a non-deterministic process algebra. In particular, the BNDC (bisimulation-based nondeducibility on compositions) property prevents attackers from affecting the availabilities of observable process actions. However, the BNDC property requires observational equivalence, making it difficult to separate the concerns for integrity and availability.

Yu and Gligor [31] develop a formal method for analyzing availability: a form of first-order temporal logic is used to specify safety and liveness constraints on the inputs and behaviors of a service, and then those constraints can be used to formally verify the availability guarantees of the service. The flexibility and expressiveness of first-order temporal logic come at a price: it is difficult to automate the verification process. The approach of formalizing and reasoning system constraints and guarantees in terms of logic resembles the rely-guarantee method [12], which was also applied to analyzing cryptographic protocols by Guttman et al. [10].

Lafrance and Mullins [14] define a semantic security property impassivity for preventing DoS attacks. Intuitively, impassivity means that low-cost actions cannot interfere with high-cost actions. In some sense, impassivity is an integrity noninterference property, if we treat low-cost as lowintegrity and high-cost as high-integrity. With the implicit assumption that high-cost actions may exhaust system resources and render a system unavailable, impassivity corresponds to one part of our notion of availability noninterference: low-integrity inputs cannot affect the availabilities of highly available outputs.

Li et al. [16] formalize the notion that highly available data does not depend on low-availability data. However, their definition is termination-insensitive [24], which makes it inappropriate to model availability noninterference.

Volpano and Smith [29] introduce the notion of termination agreement, which requires two executions indistinguishable to low-confidentiality users to both terminate or both diverge. The integrity dual of termination agreement can be viewed as a special case of the availability noninterference in which termination is treated as the only output of a program.

Language-based information flow control techniques [7, 
$24,30,11,32,22,3,33]$ can be used to enforce noninterference. But they mainly dealt with confidentiality and integrity. Our work focuses on applying the security-typed language approach to enforcing availability policies.

Myers and Liskov proposed the decentralized label model for specifying information flow policies [20]. This paper generalizes the DLM to provide a unified framework for specifying confidentiality, integrity and availability policies. The form of a combined security policy is an instance of an owned policy [5], though we give a different semantics here.

\section{Conclusions}

This paper makes three contributions. First, it proposes a way to specify availability policies as an extension to the decentralized label model, including the added expressive power of conjunctive and disjunctive principals and a new semantics for policies and labels. Second, the paper presents a simple language that can explicitly specify security policies as type annotations and has a security type system to reason about end-to-end availability policies, along with confidentiality and integrity policies. Third, the paper formally defines an end-to-end availability property in terms of program traces and shows that the security type system enforces this property. As far as we know, this is the first security type system for reasoning about availability.

An important direction for future work is to apply this static availability analysis framework to multithreaded programming models, and develop a notion of possibilistic (or probabilistic) availability noninterference.

\section{Acknowledgements}

The authors would like to thank Andrei Sabelfeld, Fred B. Schneider, Stephen Chong and Lorenzo Alvisi for their insightful suggestions and comments on this work. In addition to the anonymous reviewers, Nate Nystrom, Michael Clarkson, Kevin O’Neill and Jed Liu all helped improve this paper.

This research was supported in part by the Department of the Navy, Office of Naval Research, ONR Grant N00014-01-1-0968, and by National Science Foundation grants 0208642, 0133302, and 0430161. Andrew Myers is supported by an Alfred P. Sloan Research Fellowship. The U.S. Government is authorized to reproduce and distribute reprints for Government purposes, notwithstanding any copyright annotation thereon. The views and conclusions here are those of the authors and do not necessarily reflect the views of these sponsors.

\section{References}

[1] M. Abadi, M. Burrows, B. W. Lampson, and G. D. Plotkin. A calculus for access control in distributed systems. TOPLAS, 15(4):706-734, 1993.

[2] J. Agat. Transforming out timing leaks. In Proc. 27th ACM Symp. on Principles of Programming Languages (POPL), pages 40-53, Boston, MA, Jan. 2000.

[3] A. Banerjee and D. A. Naumann. Secure information flow and pointer confinement in a Java-like language. In IEEE Computer Security Foundations Workshop (CSFW), June 2002.

[4] M. Castro and B. Liskov. Practical Byzantine Fault Tolerance. In Proc. 3rd Symposium on Operating Systems Design and Implementation, New Orleans, LA, Feb. 1999.

[5] H. Chen and S. Chong. Owned policies for information security. In Proc. 17th IEEE Computer Security Foundations Workshop, June 2004.

[6] D. E. Denning. Cryptography and Data Security. AddisonWesley, Reading, Massachusetts, 1982.

[7] D. E. Denning and P. J. Denning. Certification of programs for secure information flow. Comm. of the ACM, 20(7):504513, July 1977.

[8] R. Focardi and R. Gorrieri. A classification of security properties for process algebras. Journal of Computer Security, 3(1):5-33, 1995.

[9] J. A. Goguen and J. Meseguer. Security policies and security models. In Proc. IEEE Symposium on Security and Privacy, pages 11-20, Apr. 1982.

[10] J. D. Guttman and et al. Trust management in strand spaces: A rely-guarantee method. In Proc. European Symposium on Programming, pages 325-339, Apr. 2004.

[11] N. Heintze and J. G. Riecke. The SLam calculus: Programming with secrecy and integrity. In Proc. 25th ACM Symp. on Principles of Programming Languages (POPL), pages 365377, San Diego, California, Jan. 1998.

[12] C. B. Jones. Tentative steps toward a development method for interfering programs. ACM Trans. Prog. Lang. Syst., 5(4):596-619, 1983.

[13] A. Juels and J. Brainard. Client puzzles: A cryptographic countermeasure against connection depletion attacks. In Proceedings of NDSS'99 (Network and Distributed System Security Symposium), pages 151-165, 1999.

[14] S. Lafrance and J. Mullins. Using admissible interference to detect denial of service vulnerabilities. In 6th International Workshop on Formal Methods, pages 1-19, July 2003.

[15] L. Lamport. Proving the correctness of multiprocess programs. IEEE Transactions on Software Engineering, SE3(2):125-143, March 1977.

[16] P. Li, Y. Mao, and S. Zdancewic. Information integrity policies. In Proceedings of the Workshop on Formal Aspects in Security and Trust, Sept. 2003.

[17] D. Malkhi and M. Reiter. Byzantine quorum systems. In Proc. of the 29th ACM Symposium on Theory of Computing, pages 569-578, El Paso, Texas, May 1997. 
[18] J. McLean. A general theory of composition for trace sets closed under selective interleaving functions. In Proc. IEEE Symposium on Security and Privacy, pages 79-93, May 1994.

[19] J. K. Millen. A resource allocation model for denial of service. In Proc. IEEE Symposium on Security and Privacy, pages 137-147, Oakland, CA, USA, May 1992.

[20] A. C. Myers and B. Liskov. A decentralized model for information flow control. In Proc. 17th ACM Symp. on Operating System Principles (SOSP), pages 129-142, Saint-Malo, France, 1997.

[21] A. C. Myers and B. Liskov. Protecting privacy using the decentralized label model. ACM Transactions on Software Engineering and Methodology, 9(4):410-442, Oct. 2000.

[22] F. Pottier and V. Simonet. Information flow inference for ML. In Proc. 29th ACM Symp. on Principles of Programming Languages (POPL), pages 319-330, 2002.

[23] A. Sabelfeld and H. Mantel. Static confidentiality enforcement for distributed programs. In Proceedings of the 9th International Static Analysis Symposium, volume 2477 of LNCS, Madrid, Spain, Sept. 2002. Springer-Verlag.

[24] A. Sabelfeld and A. Myers. Language-based informationflow security. IEEE Journal on Selected Areas in Communications, 21(1):5-19, Jan. 2003.

[25] F. B. Schneider. Implementing fault-tolerant services using the state machine approach: a tutorial. ACM Computing Surveys, 22(4):299-319, Dec. 1990.

[26] C. L. Schuba and et al. Analysis of a denial of service attack on TCP. In Proc. IEEE Symposium on Security and Privacy, pages 208-223, May 1997.

[27] T. K. Shridharbhai. Probability and Statistics with Reliability, Queuing, and Computer Science Applications. Englewood Cliffs, N.J. : Prentice-Hall, 1st edition, 1982.

[28] G. Smith and D. Volpano. Secure information flow in a multi-threaded imperative language. In Proc. 25th ACM Symp. on Principles of Programming Languages (POPL), pages 355-364, San Diego, California, Jan. 1998.

[29] D. Volpano and G. Smith. Eliminating covert flows with minimum typings. In Proc. 10th IEEE Computer Security Foundations Workshop. IEEE Computer Society Press, 1997.

[30] D. Volpano, G. Smith, and C. Irvine. A sound type system for secure flow analysis. Journal of Computer Security, 4(3):167-187, 1996.

[31] C.-F. Yu and V. D. Gligor. A specification and verification method for preventing denial of service. In Proc. IEEE Symposium on Security and Privacy, pages 187-202, Oakland, CA, USA, Apr. 1988.

[32] S. Zdancewic and A. C. Myers. Secure information flow via linear continuations. Higher Order and Symbolic Computation, 15(2-3):209-234, Sept. 2002.

[33] L. Zheng and A. C. Myers. Dynamic security labels and noninterference. In Proc. 2nd Workshop on Formal Aspects in Security and Trust, IFIP TC1 WG1.7. Springer, Aug. 2004.

[34] L. Zheng and A. C. Myers. End-to-end availability policies and noninterference. Technical Report 2005-xxxx, Cornell University Computing and Information Science, 2005. 